\title{
Quantification of Eplerenone Polymorphs by Diffuse Reflectance Infrared Fourier Transform Spectroscopy (DRIFTS)
}

\author{
PRAVEEN $\mathrm{CH}^{*}$, M. ARTHANAREESWARI, A. RAVIKIRAN and P. KAMARAJ \\ Department of Chemistry, SRM University, SRM Nagar, Kattankulathur-603203, \\ Kancheepuram District, Tamilnadu, India \\ praveen.chappa@gmail.com
}

Received 11 January 2013 / Accepted 15 February 2013

\begin{abstract}
A Quantification method for the determination of eplerenone polymorphs Form-H in FormL was developed and validated by Diffuse Reflectance Infrared Fourier Transform spectroscopy (DRIFTS) using Beer's law method. A series of standard spiked concentrations (2, 10, 22, 29, 48 and $100 \% \mathrm{w} / \mathrm{w}$ ) were prepared by accurately weighing and mixing the pure polymorphs. Specimens were prepared in potassium bromide powder at the concentration of $2 \% \mathrm{w} / \mathrm{w}$ and the spectra were collected. A linear relationship between the content of Form-H and the peak area at about $1406 \mathrm{~cm}^{-1}$, corresponding to Form-H was observed with an excellent correlation coefficient 0.9996 and standard error of prediction is 2.2279 using Beer's law method. The proposed method was found to be fast and reliable for the prediction of polymorphic content of eplerenone Form-H in Form-L in drug substance during processing and storage.
\end{abstract}

Keywords: Eplerenone, Quantification, Diffuse reflectance infrared Fourier transform spectroscopy

\section{Introduction}

Eplerenone, chemically pregn-4-ene-7,21-dicarboxylic acid, 9,11-epoxy-17-hydroxy-3-oxo, $\gamma$-lactone, methyl ester $(7 \alpha, 11 \alpha, 17 \alpha)$ represented in Figure 1, is an aldosterone receptor antagonist and is used for the treatment of pathological condition associated with hyper aldosterone including hypertension, heart failure and cirrhosis of liver. It is known that Eplerenone exists in two anhydrous crystal forms, designated ${ }^{1}$ as Form-H and Form-L.

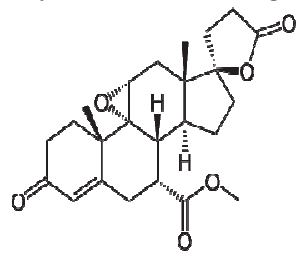

Figure 1. Molecular structure of eplerenone 
Polymorphism is a well-recognized phenomenon in the pharmaceutical industry, since different polymorphs show different stability and/or bioavailability ${ }^{2}$. A conversion from one polymorphic form to another more stable one during storage may therefore have unfavorable effects for the end user of the drug. Knowledge of the precise crystalline form of an active pharmaceutical ingredient (API) at all stages of the drug manufacturing process and the effects of storage on the formulated product are extremely important ${ }^{3}$. In order to control polymorphism in the manufacturing process of medicines, methods must be developed to differentiate and quantitate different crystal forms.

\section{Experimental}

Pure crystalline Form- $\mathrm{H}$ and $\mathrm{L}$ of eplerenone and IR grade potassium bromide samples were purchased from Sigma Company. The obtained crystal forms were characterized by X-ray powder diffraction to confirm the identity of the crystal phases by comparison to the literature.

\section{Sample preparation}

A series of standard spiked concentrations (2, 4, 10, 15, 22, 29, 40, 48, 60, 71 and 100\% $\mathrm{w} / \mathrm{w})$ were prepared by accurately weighing and mixing the pure polymorphs to a fine powder. Specimens were prepared in potassium bromide powder at the concentration of $2 \%$ w/w of drug in potassium bromide powder.

\section{Instrumentation}

\section{Powder X-ray diffractometer(PXRD) measurements}

The experiments were carried out using PANanalytical X'Pert PRO X-ray Powder Diffractometer using copper radiation $\mathrm{K}_{1}$ with $\mathrm{X}^{\prime}$ Celerator detector. The instrument was calibrated using NIST standard reference material 1976a (Corundum) for relative intensities and 640C (Silicon) for peak position. Each sample diffraction profile was collected using following setting parameters of the diffractometer: $\mathrm{X}$-Ray tube is operated at a voltage of $40 \mathrm{kV}$ and current of $30 \mathrm{~mA}$; $\mathrm{Ni}$ filtered $\mathrm{Cu} \mathrm{K \alpha} \alpha_{1}$ radiation $\left(\lambda=1.5418 \mathrm{~A}^{\circ}\right)$; scan type continues mode; scan range $2^{\circ}$ to $50^{\circ} 2$ theta ; step size $0.03^{\circ} 2$ theta; time per step 50.0 sec. Data acquisition and analysis were performed on X'pert data collector and High Score Plus software's respectively

\section{Diffuse reflectance Fourier transform infrared (DRFT-IR) measurements}

The eplerenone samples were measured using a Perkin- Elmer FT-IR spectrophotometer accompanied to a diffuse reflectance accessory. Grounded potassium bromide powder was used as the background in the measurements.

Mixtures with 2, 10, 22, 29, 48 and 100\% w/w of Form $\mathrm{H}$ were used for calibration and mixtures with 4, 15, 40, 60 and $71 \%$ w/w of Form $\mathrm{H}$ used as a validation data set. The spectral resolution used was $4 \mathrm{~cm}^{-1}$ and the number of averaging scans was 10 . The measured wave number range was from 4000 to $450 \mathrm{~cm}^{-1}$. The Sample mixtures were placed in the sample cup holders of the spectrophotometer, a cover slip was then dragged across the top of the cup to remove excess powder and smooth the sample surface. The FTIR measurements were performed on a Perkin-Elmer Spectrum One and a Perkin-Elmer DRIFTS accessory using Potassium bromide as reference material ${ }^{4-6}$.

In the present study, we investigated the use of DRIFTS to analyze the polymorphic purity of crystalline eplerenone using a specific peak of Form-H in the DRIFT spectrum, occurring at $1406 \mathrm{~cm}^{-1}$. The identities of both polymorphic forms were confirmed by comparing their X-ray diffraction (XRD) patterns Figure 2 with patent reported patterns ${ }^{1}$. 


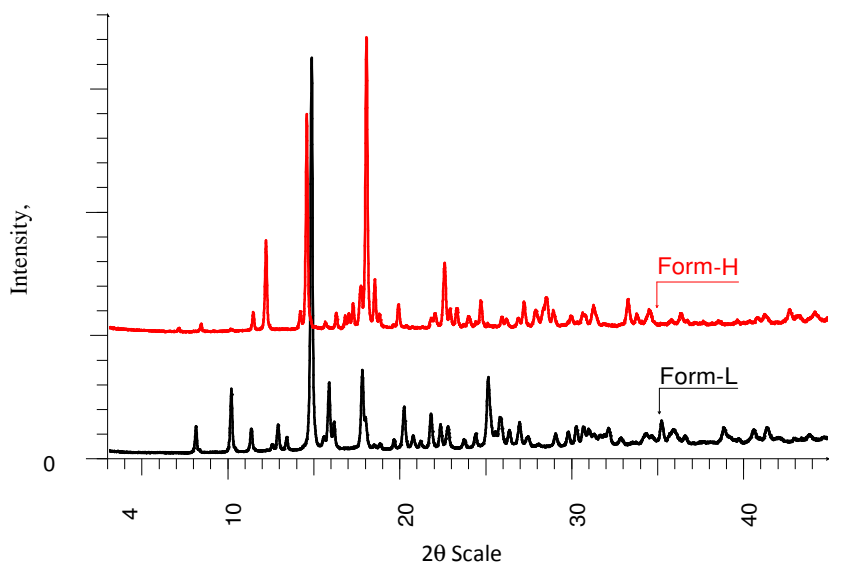

Figure 2. Powder X-ray diffraction overlay of eplerenone Forms H and Form-L

The eplerenone samples were measured using a Perkin- Elmer FT-IR spectrophotometer accompanied to a diffuse reflectance accessory ${ }^{7}$. Grounded potassium bromide powder was used as the background in the measurements. A series of standard spiked $(2,10,22,29,48$ and $100 \% \mathrm{w} / \mathrm{w}$ ) were prepared by accurately weighing and mixing the pure polymorphs. Specimens were prepared in potassium bromide powder at the concentration of $2 \% \mathrm{w} / \mathrm{w}$ of drug in potassium bromide powder ${ }^{8}$.

Mixtures with 2, 10, 22, 29, 48 and 100\% w/w of Form $\mathrm{H}$ were used for calibration and mixtures with $4,15,40,60$ and $71 \% \mathrm{w} / \mathrm{w}$ of form $\mathrm{H}$ used as a validation data set. The spectral resolution used was $4 \mathrm{~cm}^{-1}$ and the number of averaging scans was 10 . The measured wave number range was from 4000 to $450 \mathrm{~cm}^{-1}$. The Sample mixtures were placed in the sample cup holders of the spectrophotometer, a cover slip was then dragged across the top of the cup to remove excess powder and smooth the sample surface. The FTIR measurements were performed on a Perkin-Elmer Spectrum One and a Perkin-Elmer DRIFTS accessory using Potassium bromide as reference material ${ }^{4-6}$. FT- IR spectra of individual Forms represented in Figure 3 and Enlarged FT-IR Spectra of eplerenone Forms $\mathrm{H}$ at different concentrations showed in Figure 4.

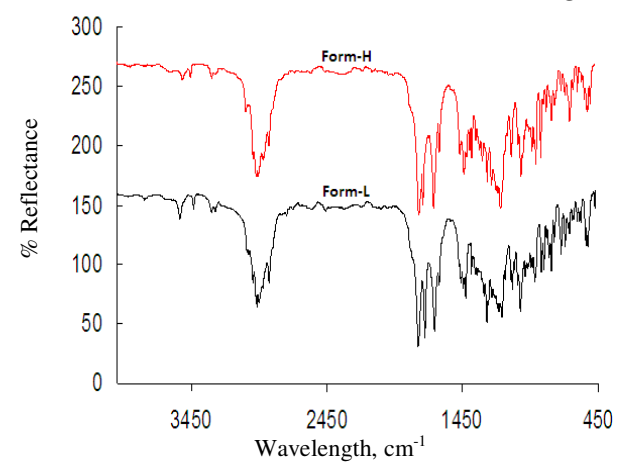

Figure 3. FT-IR spectra of eplerenone Forms $\mathrm{H}$ and Form-L

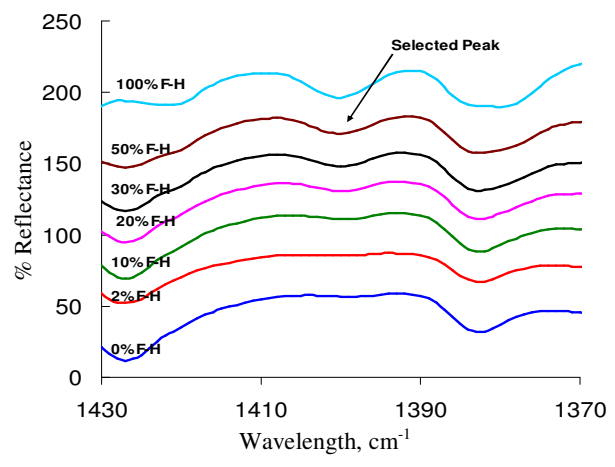

Figure 4. Enlarged FT-IR Spectra of eplerenone Forms $\mathrm{H}$ in different concentrations at $1406 \mathrm{~cm}^{-1}$ peak 
The obtained spectra were further quantitatively analyzed by the Beer's law concentration calculations using the peak area measurements. The calibration model was built using Perkin Elmer Spectrum Beer's law software. The area of the peak at about 1406 $\mathrm{cm}^{-1}$ was determined using baseline points at 1390 and $1410 \mathrm{~cm}^{-1}$. The peak area was correlated against the eplerenone Form-H concentration $(\% \mathrm{w} / \mathrm{w})$; the correlation coefficient found to be 0.9996 with a standard error of prediction 2.2279 , a linear plot of predicted values versus actual values of Form-H content is represented in Figure 5. These calibration models were subsequently validated with the external test set and the predicted values for external standards of validation are reported in Table 1 . The results demonstrated an excellent correlation and acceptable accuracy in predictions.

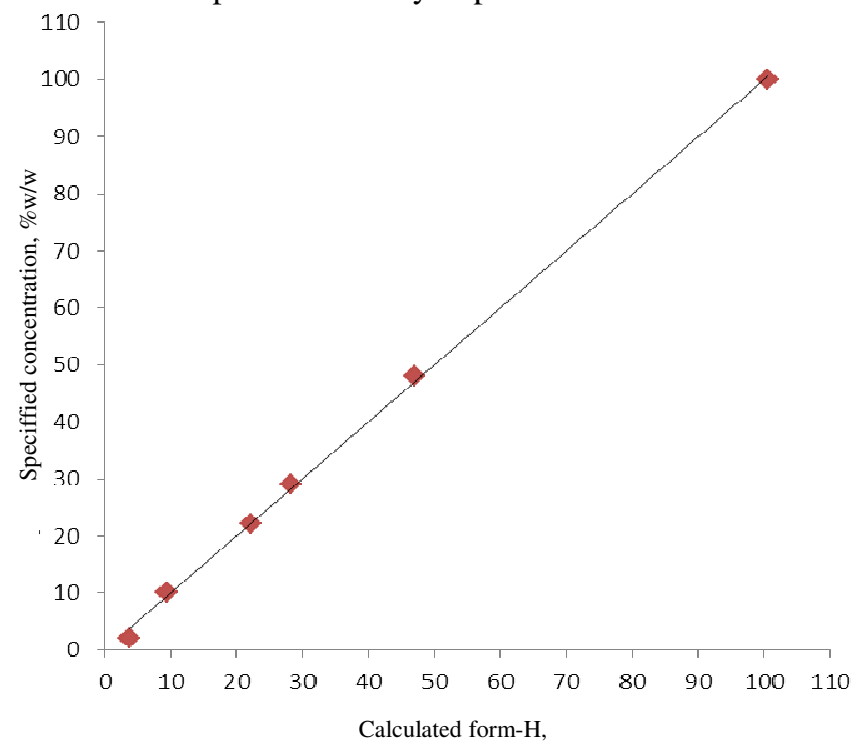

Figure 5. A plot of specified concentration versus calculated concentration of eplerenone Form-H

Table 1. Weighed (actual) and calculated composition (wt. fraction) for synthetically mixture of eplerenone Form-H in Form- $\mathrm{L}$

\begin{tabular}{ccc}
\hline $\begin{array}{c}\text { Actual spiked concentration } \\
(\% \mathrm{w} / \mathrm{w}) \text { of Form-H in Form-L }\end{array}$ & $\begin{array}{c}\text { Predicated } \\
\text { Concentrations, \%w/w }\end{array}$ & Residual \\
\hline 4.4 & 3.78 & 0.62 \\
15.0 & 15.02 & -0.02 \\
40.3 & 39.68 & 0.62 \\
60.2 & 59.36 & 0.84 \\
70.9 & 71.36 & -0.46 \\
\hline
\end{tabular}

\section{Conclusion}

Quantification of the eplerenone polymorphs based on the simple analysis of a single peak by Beer's law method using DRIFT is possible and represents a rapid, simple and easily available alternative to commonly used X-ray powder diffractometric analysis. 


\section{References}

1. Kathleen P. Barton and Thomas B. Borchardt, United States Patent Number 2002/0038021 A1 (March.28, 2002), Eplerenone crystalline form exhibiting enhanced dissolution rate.

2. Brittain $\mathrm{H} G$ (Ed), Polymorphism in pharmaceutical solids, Drugs and the pharmaceutical sciences, 95, Marcel Dekker Inc., New York, 1999.

3. Tommi Virtanen and Sirkka L Maunu, Int J Pharm., 2010, 394(1-2), 18-25.

4. Hartauer K J, Miller E S and Guillory J K, Int J Pharm., 1992, 85, 163-174.

5. Brian Connolly, Thomas W Patapoff and Y John Wang, Ana Biochem., 2010, 399, 48-57.

6. Bugay D E, Newman A W and Findlay W P, J Pharm Biomed Anal., 1996, 15, 49-61.

7. Martin E Auera and Ulrich J Griesser, J Mol Strut., 2003, 661-662, 307-317.

8. Bugay D E and Williams A C, In: Brittain H G (Ed.), Physical Characterization of Pharmaceutical Solids, Vibrational Spectroscopy, Marcel Dekker, New York, 1995, pp 59-91. 